\title{
ADVANCEMENT OF THE RHIC BEAM ABORT KICKER SYSTEM *
}

\author{
W. Zhang, L. Abrens, J. Mi, B. Oerter, J. Sandberg, D. Warburton \\ Brookhaven National Laboratory, Upton, NY 11973-5000
}

\begin{abstract}
As one of the most critical system for RHIC operation, the beam abort kicker system has to be highly available, reliable, and stable for the entire operating range. Along with the RHIC commission and operation, consistent efforis have been spend to cope with immediate issues as well as inherited design issues. Major design changes have been implemented to achieve the higher operating voltage, longer high voltage hold-off time, fast tetriggering and redundant triggering, and improved system protection, etc. Recent system test has demonstrated for the first time that both blue ring and yellow ring beam abort systems have achieved more than 24 hours hold off time at desired operating voltage. In this paper, we report on the system stanus and improvements.
\end{abstract}

\section{INTRODUCTION}

Each RHIC Beam Abort System has five high voltage modulators located inside tunnel directly connected to their corresponding magnet sections. There are two identical systems, one for each of the RHIC Blue and Yellow Ring. The kicker system charging voltage tracks the beam energy during beam injection, acceleration, and storage. Its operation range is from $4 \mathrm{kV}$ to $27 \mathrm{kV}$. At top beam energy, a pulsed current of $18 \mathrm{kA}$ per module is required to clear the circulating beams from the superconducting ring. The charging voltage is about 27 $\mathrm{kV}$ for this pulsed output current level. During beam storage, all capacitor banks of the high voltage modulators will maintain at commanded voltage level for up to ten hours until being discharged.

The discharging event is usually a normal beam abort at the end of store, or an urgent request due to any ring system pulled beam permit. Along with the effort to increase instantaneous and integrated beam luminosity, the improvement and advancement of the existing modulator systems has been continuing.

In operation, if one of the thyratrons prematurely conducts, the beam will be lost around the ring. With high energy and high intensity beam, this beam loss can quench the superconducting magnet and damage valuable and sophisticate detectors used by RHIC experiment. Like many other complicated issues, multiple factors contributed to the RHIC Abort Kicker instability. Besides the known high voltage problems there are other phenomena that seem to be related to the beam energy, beam intensity, beam loss level, and possibly beam coupling, etc. With engineering design and construction issues and physics issues tangled together, the analysis of a particular event is often inconclusive. The first step is to

* Work performed under Contract Number DE-ACO2-98CH10886 with the auspices of the US Department of Energy. solve inherited engineering issues, such as high voltage break down, thyratron reverse arcing, and to build a fast re-trigger system to reduce beam spreading in event of premature discharge.

\section{HIGH VOLTAGE HOLD-OFF IMPROVEMENT}

The high voltage modulator of the RHIC Beam Abort Kicker consists of a Pulse-Forming-Network in series with a thyratron switch. Each capacitor bank has a capacitance of $8.3 \mu \mathrm{F}$; it stores $3 \mathrm{~kJ}$ of energy at $27 \mathrm{kV}$. Its high voltage section had a history of arcing but difficult to locate. Uswally, a corona discharge would trigger the thyratron to discharge the PFN. Occasionally, a very loud sparking noise can be heard.

A series of test was performed on the prototype modulator to identify problem area. Eventually, a large conductor plate made of shatp copper sheet and several insulators with metallic inserts, hiding behind an aluminum extrusion for structure support and front panel mounting, were found to be the main problem. They were improperly designed and constructed. The removed conductor plate was badly discolored and showed clear corona marks and arcing signs along its edges. It was located right beneath the thyratron anode connection, and its corona discharge current can easily set off the thyratron. The removed insulating material and high voltage standoffs were found to have had internal discharges due to closely spaced metallic inserts from end to end. It was necessary to redesign the conductor plate and high voltage standaffs that can fit into the existing place and to eliminate the corona discharges for higher voltage hold-off.

After design modification, the prototype unit voltage hold off level was largely improved. It was DC tested at $30 \mathrm{kV}$ and $35 \mathrm{kV}$ for eight hours continuously for several days. All ten high voltage modulators in the RHIC ring were modified accordingly to gain higher voltage holdoffs. The RHIC Beam Abort Kicker high voltage modulators was tested for high voltage DC hold-off at desired operating refetence voltage of $27 \mathrm{kV}$ for more than 36 hours continuously at Yellow Ring, and for more than 60 hours continuously at the Blue Ring. The DC tests were successful, and no acing or corona discharges were observed during these tests.

\section{FAST RE-TRIGGER SYSTEM}

A temporary fast re-trigger system using kicker output current read-back signals through a multi-channel $R F$ combiner to re-trigger the trigger and delay generator was adopted for a quick fix during 2001-2002 operation. This has a delay of 2.5 us between the first and rest channels 
due to transmission time of lengthy signal and trigger cables between the service building and the modulators located in the ring. A re-trigger system with less delay and wider tesponse range was designed and implemented later during 2001-2002 operation. However, the partial beam loss was still big enough to cause experimenters concern for the detectors.

Prior to the 2003 operation, the re-rigger system together with the redundant high voltage trigger generators and power supply were moved into ring to shorter cable length caused delays. This helps reducing partial beam loss to an acceptable level to the experiments. However, the semiconductor devices used in the present version is not intended for application in the radiation area, and a new design with better noise immunity and radiation hardness is underway.

\section{SWTCH EVALUATION}

The hollow-anode thyratrons are used as main switch in the RHIC Beam Abort Kicker modulators. Two different models, CX1575C and CX3575C, are being used in operation. To reverse conduct several kilo-Amperes of current for $30 \mu \mathrm{S}$ requires a large amount of plasma, which well exceeds the capability of any hollow anode thyratrons. The thyratrons can be damaged due to reverse arcing, and their degradation of high voltage hold-off has been reported [1] [2]. Each year, several thyratrons have to be replaced. Some of them can be reconditioned and some are permanently damaged. CX $1575 \mathrm{C}$ was the version used in the original design, and the CX3575C is a larger version of $\mathrm{CX} 1575 \mathrm{C}$ with higher voltage and current capability. It performed better than CX1575C in the test stand during evaluation. [2]. Therefore, all the thyratrons in the Yellow Ring system were changed to CX3575C during 2002 operation. However, this change did not stop the misfire situation of the Yellow Beam Abort System, and two CX3575C had to be replaced soon after their installation.

Many attempts were made to stabilize the thyratrons in operation. The reservoir voltages of several thyratrons have to be reduced to lower the gas pressures in hope to lessen their sensitivities to beam operation. Although reverse arcing is a damaging factor to thyratron, but this is usually a gradual degradation of the voltage hold-off capability. The damage patterns from recently replaced thyratrons suggest the damage induced by the misfire without sufficient plasma ionization ruins thyratron much faster and drastic than under normal operation condition. The Blue Ring Beam Abort Kicker System has a much lower rate of misfiring, and the thyratrons are lasting much longer.

The premature discharge problem during this run has some association to the re-triggering system moved into the ring. The output current read-back cables bring the beam signal and other noises into the re-trigger system inputs. The large amplitudes of noises and beam signal can cause trigger misbehavior or induce output oscillation. Several modifications were made to improve its noise susceptibility, and filters were added to the thyratron grids inside high voltage modulators.

To further improve the switch performance, a new gas switch HX3002 developed by E2V has been recommended as a possible candidate for RHIC Beam Abort Kicker application. This switch is capable of high reverse current conduction. It is designed for low repetition rate, high-energy pulser applications, such as crowbar switch [3]. Figure 1 shows both CX1575C thyratron and $\mathrm{HX} 3002$ gas switch.

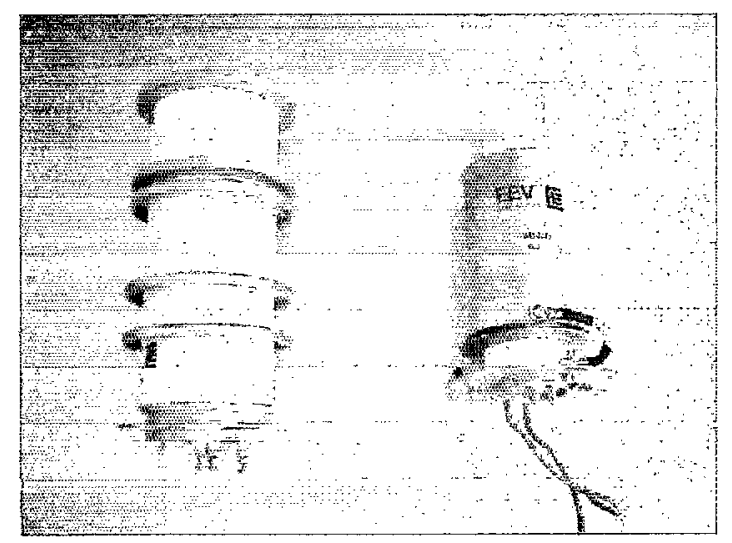

Figure 1: A CX1575C thyratron and a HX3002 gas switch

The HX3002 gas switch is rated for $38 \mathrm{kV}, 20 \mathrm{kA}$ peak forward conduction. Its reverse current capability was factory tested to $17 \mathrm{kA}$. The RHIC Beam Abort Kicker operation range is from $4 \mathrm{kV}$ to $27 \mathrm{kV}$. The required output current at $27 \mathrm{kV}$ is about $18 \mathrm{kA}$. We tested the HX3002 with a low energy pulser from $5 \mathrm{kV}$ to $35 \mathrm{kV}$, with 3500 pulses, and hi-potted it at $38 \mathrm{kV}$ for more than 20 hours after the pulse test. The full energy pulse test in the RHIC Beam Abort prototype module was performed up to $30 \mathrm{kV}$, with $5 \mathrm{kV}$ increments, for about 2000 pulses. At $30 \mathrm{kV}$, the current reached $21.69 \mathrm{kA}$ peak. The waveform obtained from this switch tube is identical to the one with thyratron. A single-shot L-C oscillation test was done at $30 \mathrm{kV}$ level, the current swing between $+/ \sim 14$ $\mathrm{kA}$, as shown in figure 2. Again, no damage or degradation was observed. This bi-direction capability is far superior to a thyratron.

It has been installed in one of the Yellow ring high voltage modulators recently to test under real operation environment. The Base mounting dimensions of hollow anode thyratron $\mathrm{CX} 1575 \mathrm{C}, \mathrm{CX} 3575 \mathrm{C}$, and $\mathrm{HX} 3002$ are identical. The HX3002 is slightly shorter than the thyratrons, therefore easy to fit in the thyratron mounting position. To reduce the chance of gas breakdown under high voltage, a double trigger pulsed ionization scheme is selected. In this method, no DC pre-ionization is provided, and is assumed to be less likely to self-conduct. A 30 Amperes current trigger is provided to drive the gas tube, and the anode delay time is around $0.7 \mu$ s. This current was found to saturate the existing high voltage isolation pulse transformer used for thyratron triggering; 
and a coaxial trigger transformer made of high voltage cable and ferrite ring was constructed to replace it.

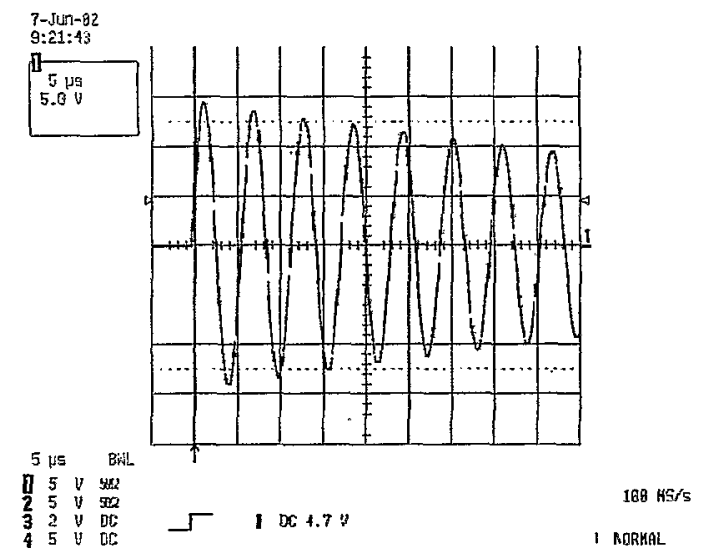

Figure 2: HX3002 oscillating current waveform at $30 \mathrm{kV}$, $+/ 14 \mathrm{kA}$ operating condition.

\section{CONCLUSION}

Consistent performance improvement has been achieved in RHIC Beam Abort Kicker systems. The future investigation will focus on the following issues:

The Blue and Yellow Ring Beam Abort Kicker System are assumed to be identical, and no apparent difference has been found. But, the Blue Ring Beam Abort Kicker System is much more stable than the Yellow one.

The beam passing through the kicker aperture couples to the kicker magnet. The kicker magnet pulse current transformers show clear beam current signals. There is a signature that can be detected on all kicker current transformers prior to each misfire. The kicker will usually respond to it in a few hundred nanoseconds to a microsecond dependant on its magnitude. The threshold of the kicker response has been increased, but with higher beam energy and higher intensity the struggle continues. This signature is common to all units, and is therefore assumed to associate with structures such as beam chamber, grounding, beam property, etc. Figure 3 shows a typical misfire situation, where a sudden change in channel 4 signal followed by the kicker current rise. In this setup the channel 4 is set at very high gain to detect the event. The currents in two other channels, with lower scope gain, and a beam bunch pick up signal are also shown. The sweep time is about $5 \mu$ s. Since all beam loss monitors in the RHIC ring around sector 9 and downstream of it are integrator type, the response time is too slow to detect it. The time resolution of vacmum pressure change sampling is in millisecond domain, there is no fast detection available to diagnose transient event in vacuum. Meanwhile, the kicker itself is the device fast enough to detect the phenomenon. Twelve PIN diodes detectors are to be installed around the beam abort kickers to assist diagnostics.

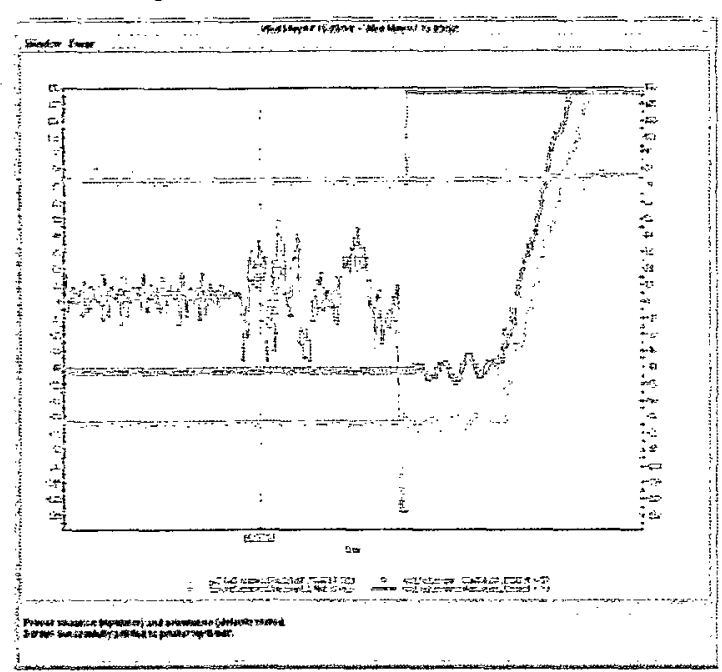

Figure 3: A typical premature discharge following a signature event detected by the pulse current transformer

To use a resonant charge power supply to charge up the capacitor banks in a few milliseconds upon receiving the beam abort command has been suggested. In this way, the main switch of the high voltage modulators will be unstressed most time during beam operation, and only under high voltage stress for a few milliseconds right before beam abort. This shall provide better operation stability and is being studied.

\section{ACKNOWLEDGEMENT}

We would like to thank Dr. H. Hahn of BNL, Dr. R. Sheldrake and Dr. C. Pirrie of E2V for their expert advice, and to Mr. J. Addessi, Mr. K. Hartmann, Mr. S. Perlstein, and Mr. R. Zapasek for their technical support and contribution.

\section{REFERENCE}

[1] H. Hahn, A. Dunbar, C. I. Pai, R. T. Sanders, N. Tsoupas, f. E. Tuozzolo, "THE RHIC BEAM ABORT KECKER SYSTEM", IEEE Proceedings of The 1999 Particle Accelerator Conference, 1999, pp. 1100-1102.

[2] H. Hahn, "Test Results CX3575C \#1460", BNL C-A internal communication, Nov, 1999.

[3] C. A. Pirrie, and C. A. Roberts, "A new Discharge Switch for Discharging high Energy Capacitor Banks", Digest of Technical papers of the Eleventh IEEE International Pulsed Power Conference, 1997, pp. 334-339. 